\title{
Modelo de gestión de mantenimiento parcial a interruptores de potencia mediante inteligencia artificial
}

\author{
Partial maintenance to high voltage breakers management \\ model using artificial intelligence \\ Israel Gondres Torné ${ }^{*} \quad$ Santiago Lajes Choy ${ }^{1} \quad$ Alfredo del Castillo Serpa ${ }^{2}$ \\ Recibido 7 de diciembre de 2016, aceptado 1 de agosto de 2017 \\ Received: December 7, 2016 Accepted: August 1, 2017
}

\begin{abstract}
RESUMEN
En este trabajo se propone un modelo para la gestión del mantenimiento parcial a interruptores de potencia, teniendo en cuenta la integración de diferentes variables que presentan cierto grado de incertidumbre (inspección visual, datos históricos, desgaste de los contactos principales) mediante la herramienta de la teoría difusa de los conjuntos aproximados, de modo que sea la base para la conformación de un nuevo modelo de gestión de mantenimiento. El modelo está compuesto de cuatro subsistemas, el subsistema contextual operacional, el subsistema experiencial perceptivo, el subsistema de diagnóstico del estado del interruptor, y finalmente otro subsistema que se encargará de precisar la toma de decisiones. Todos los subsistemas se interrelacionan dialécticamente entre sí. Además se presentan las regularidades, fundamentos y pasos del modelo propuesto de forma tal que pueda implementarse en cualquier subestación que cuente con los datos necesarios.
\end{abstract}

Palabras clave: Gestión de mantenimiento, inteligencia artificial, interruptores de potencia, modelo de mantenimiento.

\begin{abstract}
In this paper, a model for the management of partial maintenance to high voltage breakers is proposed, regarding the integration of different variables that have some degree of uncertainty (visual inspection, historical data, wear of the main contacts) through the Fuzzy Rough Sets Theory tool. Therefore, that is the basis for the creation of a new maintenance management model. The model consists of four subsystems: operational contextual subsystem, experiential perceptual subsystem, high voltage breaker diagnosis subsystem, and finally, another subsystem that is responsible for making accurate decisions. All subsystems are dialectically interrelated. Moreover, the regularities, fundamentals, and steps of the proposed model are presented in a way that can be implemented in any substation that has the necessary data.
\end{abstract}

Keywords: Artificial intelligence, high voltage breaker, maintenance management, maintenance model.

\footnotetext{
1 Departamento de Ingeniería Eléctrica. Universidad de Camagüey “Ignacio Agramonte Loynaz”. Circunvalación Norte e/ Camino Viejo de Nuevitas y Ave. Ignacio Agramonte C.P. 74650 Camagüey, Cuba. E-mail: israel.gondres@ reduc.edu.cu; igondrest@ gmail.com; santiago.lajes@ reduc.edu.cu

2 Centro de Estudios de Matemática. Universidad Tecnológica de la Habana “José Antonio Echeverría”. Calle 14, No 11901119 y 129. Marianao. La Habana, Cuba. E-mail: acastillo@cemat.cujae.edu.cu

* Autor de correspondencia.
} 


\section{INTRODUCCIÓN}

La necesidad del mantenimiento de los interruptores de potencia no es obvia y aumenta con los años a medida que los sistemas de transmisión se expanden y transportan mayores cantidades de energía a mayores distancias, de ahí que sea de vital importancia predecir su estado. A medida que las tecnologías avanzan, también se hace necesario un nuevo enfoque en el mantenimiento, por ello es necesario contar con un modelo de gestión del mantenimiento a interruptores de potencia que logre interactuar con las diferentes variables que se presentan desde todos los puntos de vista [1].

Primeramente se parte del concepto de modelo, el que se ha representado como una forma perfectible e idealizada de una entidad física o de un fenómeno físico, que se elabora en el ámbito científico ante la necesidad de resolver un problema de investigación. A la luz de una cierta teoría, y teniendo en cuenta las herramientas experimentales y conceptuales disponibles, el investigador decide cuáles han de ser las variables a incorporar en el modelo y cuáles merecen el calificativo de "despreciables". De este modo, se va construyendo una representación que guarda una relación de analogía con el sistema real en estudio [2].

Aunque en los textos frecuentemente se llama modelos a las teorías, se considera más adecuado decir que las teorías suponen modelos y que estos modelos, y no las teorías mismas, es lo que se supone que representan los fundamentos de las teorías. En ese sentido, una teoría se refiere a un sistema o a una clase de sistemas, y los modelos representan esos sistemas. Por su parte, Adúriz describe a las teorías como familias de modelos e hipótesis que se vinculan con los fenómenos; la relación entre las declaraciones científicas y el mundo al que aluden no es directa, sino que está mediada por el correspondiente modelo [3].

El ajuste entre el modelo y la realidad se evalúa tomando en cuenta solamente "aquellos aspectos del mundo que los modelos intentan capturar", ya que la construcción de modelos conlleva, en sí misma, el proceso de "simplificación" de cada situación en estudio, la idealización y la aproximación que son típicas de las ciencias empíricas [3].

Los constructos abstractos (modelos) así elaborados, son solo algunos de los componentes del hecho real que representan. Un cierto sistema real puede ser, entonces, representado por más de un modelo y la preferencia por uno de ellos estará determinada por los propósitos del estudio y por las condiciones de su realización [4].

El tipo de correspondencia entre un modelo y una porción de la realidad no es, entonces, recíproca "uno a uno"; pensemos, por ejemplo, en las diversas maneras en las que puede ser representado un átomo, todas ellas válidas para diferentes propósitos, desde la versión más sencilla del inicial modelo de Demócrito, el de Thomson, el de Dalton, el de Rutherford, o el de Bohr hasta las más sofisticadas que se utilizan en los estudios de Física Cuántica [5].

En razón de lo antes expuesto, los autores citados hablan de los "múltiples modelos" que son válidos para representar una entidad física o un proceso físico. Esta multiplicidad de modelos válidos para un mismo sector de la realidad se relaciona con el carácter de sistema conceptual del modelo, como representación idealizada de una clase de objetos, que ofrece un mapa de un sector de la realidad, actuando como mediador entre ella y los enunciados científicos $[3,6]$.

Según el Instituto de Ingenieros Eléctricos y Electrónicos (IEEE por sus siglas en inglés) el mantenimiento no es más que el acto de preservar o mantener las condiciones de un equipo para su correcta operación; o sea, la combinación de todas las acciones técnicas y administrativas que incluyen acciones de supervisión, que puedan mantener o restaurar el estado para el que cumple con las funciones afines a su desempeño un equipo o sistema determinado [7].

El objetivo del mantenimiento de un interruptor, es asegurar la máxima confiabilidad, disponibilidad y rentabilidad del interruptor para que cumpla con sus funciones operativas nominales, previniendo o corrigiendo, cuando sea necesario, condiciones que pongan en riesgo la operación del mismo y de la instalación a la que está asociado, así como a los equipos e instalaciones vecinas [8].

\section{ANÁLISIS TEÓRICO}

La operación confiable de un sistema hace necesario conocer, por un lado, las tasas de falla, los tiempos 
de reparación, el costo de las reparaciones y los equipos propensos a fallas; $y$, por otro, se requiere conocer los tiempos de indisponibilidad por inspecciones, mantenimientos programados y sus costos asociados [9-10-11].

En ello juega un papel importante la confiabilidad de las subestaciones y dentro de ellas, los interruptores de potencia, que se expresan mediante índices de confiabilidad, los que cuantifican la calidad del servicio que presenta el sistema eléctrico en general. Dichos índices deben ser lo suficientemente consistentes y sensibles para diferenciar varias alternativas y asegurar la operación del sistema de forma tal que garantice su operación confiable y de los elementos asociados al mismo [12].

Los principales índices de confiabilidad están interrelacionados con la gestión del mantenimiento de cualquier activo, dependiendo del tipo y con qué frecuencia ocurren las fallas, la gravedad de las consecuencias y las estrategias de mantenimiento que son adecuadas para prevenir tales fallas [13]. De ahí parte el Mantenimiento Centrado en la Confiabilidad, (RCM por sus siglas en inglés), el que se considera por varios autores como una de las filosofías mundiales del mantenimiento pues contribuye a aplicar nuevos conceptos y categorías en la "actividad" ya compleja del mantenimiento, en este caso es referido al análisis de criticidad, con el fin de evaluar la gravedad de los fallos [14].

Por lo tanto, si se integran los elementos de la confiabilidad operacional dada por el análisis de criticidad y el desgaste de los contactos debido a las corrientes que circulan por medio de ellos, las mediciones de parámetros eléctricos que definen el estado del interruptor de potencia, así como las revisiones visuales y la inteligencia artificial, se está en condiciones de formular una nueva concepción del tratamiento a la gestión del mantenimiento en interruptores de potencia. A continuación se abordan las bases de dicho modelo.

Fundamentos teóricos del modelo de gestión de mantenimiento parcial a interruptores de potencia Existe una paradoja entre la certidumbre de que todas las máquinas y equipos fabricados por una firma tienen las mismas respuestas y la incertidumbre, así como la inestabilidad del funcionamiento real, máxime cuando está mediado por el hombre. Este efecto ha sido sembrado bajo la lógica formal de Aristóteles [15], cuyos principios básicos son:

- Principio de identidad: un objeto solo es igual a sí mismo;

- Principio de no contradicción: un objeto no puede estar en un conjunto y en su complementario;

- Principio del tercero excluido: un objeto solo puede estar en un conjunto o en su complemento, y no hay otra posibilidad.

Haciendo un análisis epistemológico, se observa que la realidad está mediada por lo difuso, en donde algo específico hoy, no es igual a lo mismo mañana, ni es igual a sus semejantes; pero aún más, quien opera hoy, no operará igual mañana y todos los individuos operan distinto; esto nos lleva a establecer que el pensamiento lineal se debe cambiar por uno no lineal; es decir, un nuevo modo de conocer la realidad. Es preciso hacer notar que un conjunto difuso no cumple los principios aristotélicos segundo y tercero, debido a que un elemento de un conjunto difuso puede pertenecer y no pertenecer a la vez a un mismo conjunto, simplemente porque los criterios de pertenencia no son nítidos [16].

Si se toma en cuenta al interruptor, al ser un organismo no autónomo, el producto de su funcionamiento es diferente de él mismo y, por extensión, los cambios que pueda sufrir sin alterar su identidad están subordinados a la producción de algo diferente (entre otros el mantenimiento). Este hecho caracteriza una situación de no autonomía, por lo que cada cambio queda marcado en su historia y desde luego, influenciará en su funcionamiento, por lo que es necesario tenerlo en cuenta.

En consecuencia, a continuación se enuncian los siguientes fundamentos del modelo de gestión del mantenimiento parcial:

1. La subjetividad de la gestión del mantenimiento,

2. La historia del funcionamiento del interruptor,

3. El desgaste de los contactos,

4. La relación contextual del sistema interruptorhombre,

5. Lo difuso de las revisiones visuales, $\mathrm{y}$

6. La incertidumbre en la medición de las variables.

La modelación de la gestión del mantenimiento parcial se efectúa mediante el método estructural 
funcional, basado en cuatro subsistemas cuya interrelación dialéctica se ilustra en la Figura 1.

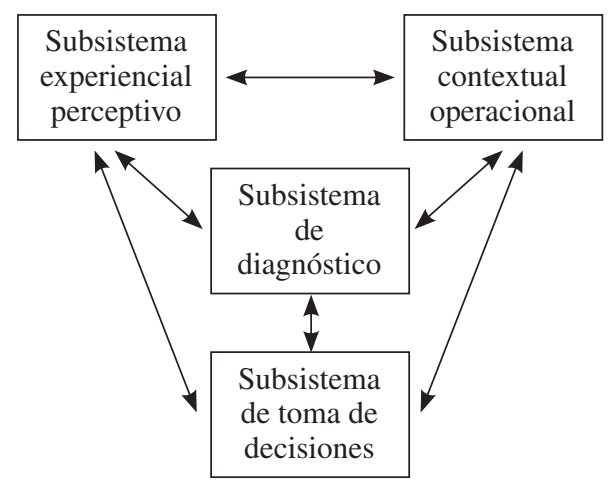

Figura 1. Modelo de gestión del mantenimiento parcial a interruptores de potencia.

El modelo está formado por cuatro subsistemas que se interrelacionan dialécticamente: i) El subsistema contextual operacional, donde se integran tanto la situación actual del interruptor por el grado de desgaste en los contactos, la confiabilidad y la historia de su funcionamiento, que deben dar una idea general de su estado a partir de datos reales; ii) El subsistema experiencial perceptivo, el que brinda el nivel de signos (subjetivos) devenidos de la experiencia y las revisiones visuales del profesional; iii) El subsistema de diagnóstico del estado del interruptor, que es una especie de síntesis de los dos primeros subsistemas; y finalmente, iv) El subsistema de toma de decisiones mediante el uso de la teoría difusa de los conjuntos aproximados.

Por tanto, el principio fundamental del modelo propuesto de la gestión del mantenimiento parcial a interruptores de potencia se determina a partir de la integración de los resultados de un diagnóstico formado por la inspección visual, los datos históricos, la confiabilidad y el posible desgaste en los contactos.

\section{Regularidades del modelo de gestión de mantenimiento propuesto}

Las regularidades del modelo propuesto están dadas en:

- El subsistema de diagnóstico para la gestión del mantenimiento parcial a interruptores de potencia es la síntesis del subsistema contextual operacional y el subsistema experiencial perceptivo.

- El subsistema de toma de decisiones para la gestión del mantenimiento parcial a interruptores de potencia, se implementa mediante un sistema experto debido al carácter difuso y la incertidumbre propia del sistema; y se basa en la Teoría Difusa de los Conjuntos Aproximados.

La función principal de este modelo de gestión del mantenimiento parcial a interruptores de potencia mediante teoría difusa de los conjuntos aproximados, es alertar cuándo un interruptor de potencia necesita mantenimiento, evitando de esta forma la ocurrencia de fallas en el sistema eléctrico y el aumento de los costos en los mismos, tratándolo como un problema de clasificación binaria [17]; es decir, dadas las entradas de las variables, se debe decidir de manera inteligente si el interruptor necesita mantenimiento (Clase Positiva) o no lo necesita (Clase Negativa).

En el desarrollo de la investigación que dio lugar a la presente propuesta, se realizaron numerosas observaciones a varios interruptores de SF6. En cada caso, un equipo de expertos determinó si era necesario o no dar mantenimiento al interruptor, por lo que se obtuvo un conjunto de datos que tiene dentro de sus características el hecho de que una clase posee muchos menos ejemplos que la otra, por lo que debido a las características observadas en los datos es un problema que se conoce dentro de la Minería de Datos como datos desbalanceados [18].

Por tanto, no es factible el uso de redes neuronales o neurodifusas, ya que estos clasificadores no pueden actuar sobre conjuntos de datos desbalanceados [19]. Por ello, se propone la integración de la lógica difusa con los conjuntos aproximados para obtener un modelo mediante teoría difusa de los conjuntos aproximados y así determinar la variable requerimiento de mantenimiento de un interruptor de potencia.

\section{Pasos del modelo de gestión de mantenimiento propuesto}

Los pasos del modelo propuesto son los siguientes:

1. Preparación de los datos a utilizar en la base de conocimientos.

- Identificación de las variables a utilizar.

- Realización de las mediciones de cada variable.

- Determinación por parte de expertos para cada una de las mediciones si necesita mantenimiento.

2. Determinación de las características de la base de conocimientos atendiendo al número de instancias en cada clase. 
3. Elección del clasificador que se usará en la aplicación.

4. Elección del algoritmo de preprocesamiento que se usará en la aplicación.

\section{RESULTADOS}

La estrategia a seguir para la gestión de mantenimiento parcial de los interruptores de potencia en una subestación determinada consta de los siguientes pasos [20]:

\section{Caracterización de la subestación donde se} encuentran los interruptores de potencia para la obtención de los datos necesarios, donde se incluyen:

a) Datos nominales de los interruptores incluyendo la gráfica de la curva típica del desgaste y ubicación en el esquema monolineal.

b) Datos de las cargas, líneas, transformadores y generadores asociados a los interruptores.

c) Datos de las revisiones visuales a los interruptores evaluadas entre 1 y 10 .

d) Valores de las pruebas de funcionamiento.

e) Cantidad de operaciones a corriente nominal y de cortocircuito.

f) Datos necesarios para el análisis de la confiabilidad operacional.

Estos datos pueden ser obtenidos a partir de los registros históricos de las subestaciones, o bien, de trabajos previos de obtención de los mismos.
2. Construcción de la base de conocimientos de acuerdo con las diferentes variables a analizar, las mismas estarán dadas por:

a) Determinación de la confiabilidad operacional mediante análisis de criticidad y expresándolo en Alta, Media o Baja [21].

b) Determinación del desgaste de los contactos de los interruptores de potencia de acuerdo con la curva típica de desgaste y la cantidad de operaciones a corriente nominal y de cortocircuito expresándolo en Alto, Medio o Bajo.

c) Obtención de valores nominales de las revisiones visuales entre 1 y 10 de los parámetros analizados (terminales primarios, porcelanas, calefacción, estado de los gabinetes, aterramiento, borneras, control general).

d) Medición y obtención de los valores reales de diferentes parámetros eléctricos (aislamiento de cámara, aislamiento apoyo, aislamiento total, resistencia de contacto, presión SF6, resistencia bobinas).

\section{Determinación de la necesidad del man-} tenimiento en el interruptor de potencia:

a) Preprocesamiento de los datos mediante la utilización del algoritmo SMOTE-FRST-2T [22, 23].

b) Clasificación de los datos mediante el árbol de decisión C4.5.

c) Resultados (necesidad o no de mantenimiento).

El diagrama de bloques del algoritmo general para la aplicación del modelo propuesto se muestra en la Figura 2.

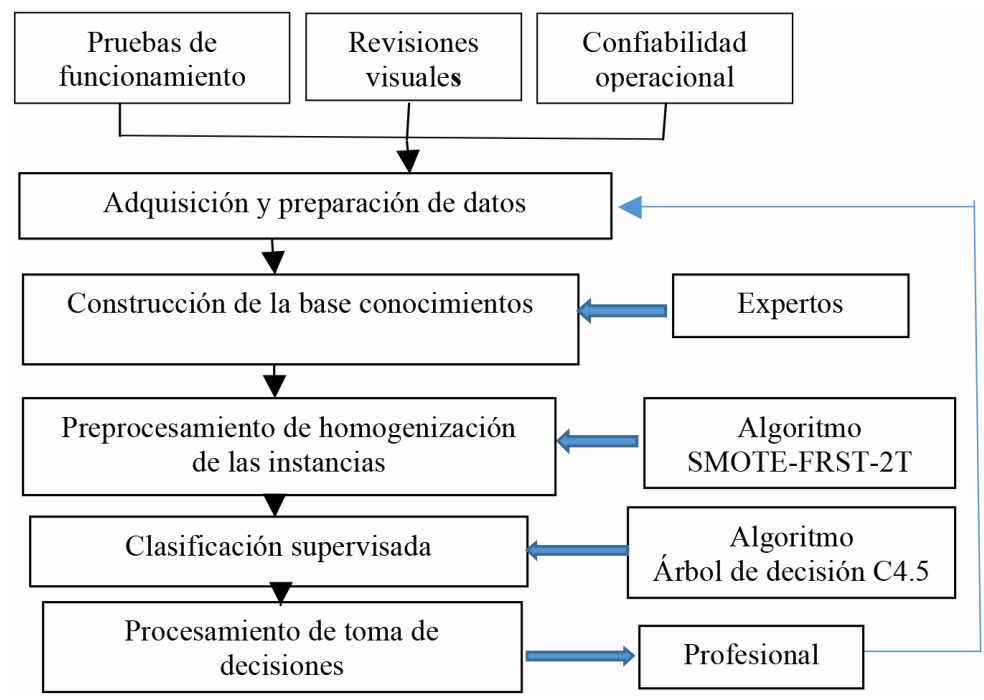

Figura 2. Diagrama de bloques de la aplicación del modelo propuesto. 


\section{CONCLUSIONES}

Se demuestra que existen potencialidades en la gestión del mantenimiento parcial a interruptores de potencia que no se explotan al máximo, tanto en la cantidad de variables o parámetros a tener en cuenta, así como en el uso de las técnicas de inteligencia artificial que han avanzado considerablemente en los últimos años, en específico las que se combinan entre sí.

Al complementar la metodología de conjuntos aproximados con las propiedades de los conjuntos difusos se puede lograr una clasificación precisa, identificando las variables que presentan deterioro así como tomar decisiones en la ejecución del mantenimiento de forma acertada.

\section{REFERENCIAS}

[1] R. D., Garzon. "High voltage circuit breakers. Design and applications". Marcel Dekker, Inc. Second Edition. New York, USA, pp. 473. 2002. ISBN: 0-8247-0799-0.

[2] J. Samaja. Epistemología y Metodología. Buenos Aires, Argentina: Eudeba. 2004. ISBN 950-23-0931-6.

[3] A. Adúriz-Bravo. A 'Semantic' view of scientific models for science education. Science \& Education. Vol. 22. Issue 7, pp. 15931611. 2013. URL: http://www. link.springer. com/article/10.1007/s11191-011-9431-7. Fecha de Consulta: 24 de enero de 2014. ISSN: 1573-1901. http://dx.doi.org/10.1007/ s11191-011-9431-7

[4] L. Grosslight, C. Unger y E. Jay. "Understanding Models and their Use in Science: Conceptions of Middle and High School Students and Experts". Journal of Research in Science Teaching. Vol. 28. N ${ }^{\circ}$ 9, pp. 799822. 1991. URL: http:// http://onlinelibrary. wiley.com/doi/10.1002/tea.v28:9/issuetoc. Fecha de Consulta: 05 de diciembre de 2016. ISSN: 1098-2736. http://dx.doi.org/10.1002/ tea.3660280907

[5] L. Valles. A los 100 años del modelo del átomo de Bohr. Revista de la Sociedad Química del Perú. Vol. 79, N² 2,pp. 97-98. 2013. Perú. URL: http://www.scielo.org.pe/scielo.php?script=sci_ arttext\&pid=S1810-634X2013000200001\& lng=es\&nrm=iso. Fecha de Consulta: 25 de abril de 2017. ISSN: 1810-634X.
[6] A. Chalmers. ¿Qué es esa cosa llamada ciencia? una valoración de la naturaleza y el estatuto de la ciencia y sus métodos (2da Edición ed.). 2011, México: Siglo XXI Editores, S.A. de C.V. ISBN 968-23-1516-6.

[7] IEEE Std 3007.2. IEEE Recommended Practice for the Maintenance of Industrial and Commercial Power Systems. New York: IEEE, 2010. pp. 1-56. E-ISBN: 978-0-7381-6452-6.

[8] T. Krontiris and G. Balzer. Assessing the effect of maintenance on high-voltage circuit breaker reliability. IEEE 11th International Conference on Probabilistic Methods Applied to Power Systems, pp. 761-766. Singapore. IEEE Conference Publications, 2010. ISBN: 978-1-4244-5721-2. http:// dx.doi.org/10.1109/PMAPS.2010.5528408 CIGRÉ Working Group 13.06, "Final report of the second international enquiry on high voltage circuit-breaker failures and defects in service", Int. Council Large Elect. Syst. (CIGRÉ), Paris, France, Tech. Brochure 83, 1994.

[10] CIGRÉ Working Group A3.06, "Final report of the 2004-2007 international enquiry on reliability of high voltage equipment, Parts 1-6", Int. Council Large Electric Systems (CIGRÉ), Paris, France, Tech. Brochures 509-514, 2012.

[11] IEEE STD C37.10. IEEE Guide for Investigation, Analysis, and Reporting of Power Circuit Breaker Failures. New York: IEEE Power \& Energy Society. 2011.

[12] T.M Lindquist, L. Bertling, R. Eriksson. "Circuit breaker failure data and reliability modelling". IET Generation, Transmission \& Distribution, Vol. 2, Issue 6, pp. 813-820. 2008. URL: http://www.ietdl.org. Fecha de Consulta: 01 de mayo de 2011. ISSN: 1751-8687. http://dx.doi.org/10.1049/ iet-gtd:20080127.

[13] P. Viveros, R. Stegmaier, F. Kristjanpoller, L. Barbera y A. Crespo. Propuesta de un modelo de gestión de mantenimiento y sus principales herramientas de apoyo. Ingeniare. Revista Chilena de Ingeniería. Vol. 21, $\mathrm{N}^{\circ} 1$, pp. 125138. 2013. Chile. URL: http://www.scielo. cl/scielo.php?script=sci_arttext\&pid=S071833052013000100011\&lng=es\&nrm=iso. Fecha de consulta: 1 de diciembre de 2016. 
ISSN: 0718-3305. http://dx.doi.org/10.4067/ S0718-33052013000 100011.

[14] Z. Johnson. Improving criticality analysis methods to influence RCM. Proceedings Annual Reliability and Maintainability Symposium (RAMS), pp. 1-3. Orlando, FL. IEEE Conference Publications, 2013. ISBN: 978-1-4673-4711-2. http://dx.doi. org/10.1109/RAMS.2013.6517699

[15] M. Correia. La actualidad de la lógica de Aristóteles. Revista de Filosofía. Vol. 62, pp. 139-150. 2006. Chile. URL: http:// www.scielo.cl/scielo.php?script $=$ sci_ arttext\&pid=S0718-43602006000100009\&ln $\mathrm{g}=$ es\&nrm=iso Fecha de Consulta: 25 de abril de 2017. ISSN: 0718-4360. http://dx.doi. org/10.4067/S0718-43602006000100009

[16] C. Crespo, R. M. Farfán y J. Lezama. Algunas características de las argumentaciones y la matemática en escenarios sin influencia aristotélica. Revista Latinoamericana de Investigación en Matemática Educativa. Vol. 12, No 1, pp. 29-66. 2009. México. URL: http://www.scielo.org.mx/scielo. php? script $=$ sci_arttext $\&$ pid $=S 1665$ $24362009000100003 \& \operatorname{lng}=e s \& n r m=i s o$ Fecha de Consulta: 25 de abril de 2017. ISSN 2007-6819.

[17] C. You-Shyang, C. Ching-Hsue and C. DaRen. A fuzzy-based rough sets classifier for forecasting quarterly PGR in the stock market (PART I). International Journal of Innovative Computing, Information and Control ICIC. Volume 7, Number 2, pp. 555-569. 2011. URL: http://www.ijicic.org/09-0931-2-1. pdf. Fecha de consulta: 19 de abril de 2013. ISSN: 1349-4198.

[18] Q. Hu, L. Zhang, S. An, D. Zhang, and D. Yu. On Robust Fuzzy Rough Set Models. IEEE Transactions on Fuzzy Systems, Vol. 20, No 4, pp. 636-651. August, 2012. ISSN 0718-3305. URL: http://ieeexplore.ieee. org. Fecha de consulta: 13 de junio de 2013. DOI: 10 10.1109/TFUZZ.2011.2181180.

[19] G. Peng, S. Tang and Y. Zhang. Imbalanced data processing based on adaptive composite sampling algorithm in the application of High Voltage Circuit Breaker maintenance. 35th Chinese Control Conference (CCC), pp. 313-317. Chengdu. IEEE Conference Publications, 2016. URL:http://ieeexplore. ieee.org/stamp/stamp.jsp?tp=\&arnumbe $r=7553102 \&$ isnumber $=7553055$ Fecha de Consulta: 25 de abril de 2017. ISSN: 1934-1768. http://dx.doi.org/10.1109/ ChiCC.2016.7553102

[20] I. Gondres. Gestión del mantenimiento parcial a interruptores de potencia mediante teoría difusa de los conjuntos aproximados. Tesis de Doctorado en Ciencias Técnicas. Universidad de Camagüey "Ignacio Agramonte Loynaz". Camagüey. Cuba. 2015.

[21] I. Gondres, S. Lajes y A. del Castillo. Evaluación de la confiabilidad en interruptores de potencia mediante la norma Norsok Z-013. Ingeniare. Revista Chilena de Ingeniería. Vol. 24, $\mathrm{N}^{\circ} 2$, pp. 198-205. 2016. Chile. URL: http://www.scielo.cl/ scielo.php?script=sci_arttext\&pid=S0718$33052016000200003 \& \operatorname{lng}=\mathrm{es} \& \mathrm{nrm}=$ iso Fecha de consulta: 5 de diciembre de 2016. ISSN: 0718-3305. http://dx.doi.org/10.4067/ S0718-33052016000200003.

[22] E. Ramentol, I. Gondres, S. Lajes, R. Bello, Y. Caballero, C. Cornelis y F. Herrera. Fuzzy-rough imbalanced learning for the diagnosis of High Voltage Circuit Breaker maintenance: The SMOTE-FRST-2T algorithm. Engineering Applications of Artificial Intelligence. Vol. 48, pp. 134-139. 2016. URL: http:// www.sciencedirect.com/ science/article/pii/S0952197615002389. Fecha de consulta: 5 de diciembre de 2016. ISSN: 0952-1976. http://dx.doi.org/10.1016/j. engappai.2015.10.009.

[23] Y. Qu, C. Shang, Q. Shen, N. M. Parthaláin and $\mathrm{W}$. Wu. Kernel-based fuzzy-rough nearest neighbor classification. IEEE International Conference on Fuzzy Systems (FUZZ), pp. 1523-1529. Taipei. IEEE Conference Publications, 2011. ISBN: 978-1-4244-7315-1. 